Kompass

Pneumologie

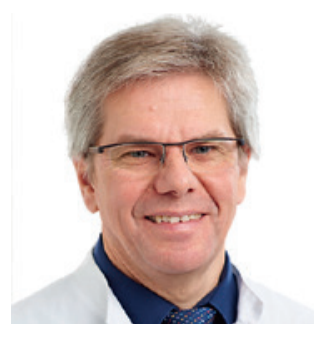

Winfried J. Randerath

Krankenhaus Bethanien gGmbH,

Klinik für Pneumologie und Allergologie,

Solingen, Deutschland

Liebe Leserinnen und Leser unseres Kompasses Pneumologie,

ich kann es fast nicht glauben, aber tatsächlich geht der Kompass Pneumologie in den 10. Jahrgang. Noch immer sind Idee und Konzept der Zeitschrift für mich ganz faszinierend und spannend: Wie können wir wichtige Publikationen einer breiten Öffentlichkeit nahebringen, die Texte analysieren, kritisch kommentieren und somit das Praxisrelevante herausarbeiten? Wie können wir junge Ärztinnen und Ärzte für das spannende Fach Pneumologie interessieren oder sie darin bestärken, innen Hinweise zum Berufsalltag geben? Wie können wir Schlaglichter auf die rasante Entwicklung im breiten Spektrum der Pneumologie werfen?

Ganz im Mittelpunkt dieser Arbeit stehen dabei natürlich die große Zahl ausgewiesener Spezialistinnen und Spezialisten von der Arbeitsmedizin bis zur Infektiologie, von der Bronchoskopie bis zur Schlafmedizin, von kardiorespiratorischen Interaktionen bis zur Intensivmedizin. Seitdem das erste Heft im März 2013 erschienen ist, haben sie in 277 Wissenstransfers für uns das ausgesucht, was innen in der Literatur besonders wichtig erschien. Nach einem vorsichtigen Beginn mit zwei Ausgaben im Jahr sind wir nun bei sechs Heften in einer Auflage von aktuell 3000 Exemplaren. Unser Expertenboard ist von 9 auf 31 ange-

\title{
10. Jahrgang Kompass Pneumologie - Spiegel der Entwicklung unseres Fachs
}

wachsen. Dies zeigt, wie breit und differenziert das Spektrum unseres Faches ist. Jede Ausgabe hat ihren besonderen Schwerpunkt, ihren Fokus, der - wie ich finde - sehr gut die Entwicklung der Pneumologie in der letzten Dekade widerspiegelt. So waren die häufigsten Fokusthemen die pneumologische Onkologie, die interstitiellen Lungenerkrankungen, aber auch Asthma und pneumologische Rehabilitation. Wir durften die wirklich beeindruckenden Fortschritte in Diagnostik und personalisierter Therapie beim Lungenkarzinom, aber auch die neuen pharmakologischen Möglichkeiten bei Lungenfibrosen und pulmonaler Hypertonie darstellen. In der Rehabilitation haben gerade deutsche Zentren zur wissenschaftlichen Weiterentwicklung erheblich beigetragen. Nach meinem persönlichen Eindruck war gerade diese Dekade von einer ganz dramatischen Entwicklung gekennzeichnet. Nicht zuletzt sei hier der Stellenwert der pneumologischen Intensiv-, Beatmungs- und Schlafmedizin erwähnt. Zum einen hat das sich Weaning von der Langzeitbeatmung zu einem besonderen Merkmal unseres Faches entwickelt. Zum anderen hat die SARS-CoV-2-Pandemie schon jetzt mehr als deutlich gemacht, welche Rolle die pneumologische Intensivmedizin mit dem gesamten Spektrum von nicht invasiver Beatmung bis hin zur ECMOTherapie spielt. Dies alles in den 10 Jahrgän- gen mitzuerleben - und vielleicht auch zu rekapitulieren - beeindruckt sehr. Kompass Pneumologie ist damit auch ein Stück Spiegel der Entwicklung unseres Faches geworden.

Wirklich von ganzem Herzen möchte ich daher allen danken, die über so lange Zeit an der Zeitschrift mitgearbeitet haben und sich weiter dafür einsetzen: Den Expertinnen und Experten unseres Fachbeirates und Dr. Matthias Raspe, der bis Ende 2019 die Rubrik Campus koordiniert hat. Ein besonderes Dankeschön aber auch an Patrick Welge, der den Kompass Pneumologie in den letzten Jahren redaktionell begleitet sowie an Christine Schiller, die maßgeblich am Aufbau und an der Konzeption der Zeitschrift beteiligt war. Ebenso gebührt Andreas Beckmann, Olga Denisow, Sibylle Gross, Anna Henschel (ehem. Piepiorka) und Katharina Treyer, den Sponsoren, die das Heft möglich machen, und dem Karger Verlag großer Dank.

Bleiben Sie, liebe Leserinnen und Leser, uns und dem Kompass Pneumologie gewogen.

Ihr

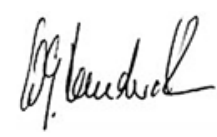

Prof. Dr. Winfried J. Randerath 